\title{
SIMULATION OF ADAPTIVE CONTROL OF CONTINUOUS STIRRED TANK REACTOR
}

\author{
Vojtesek, J. \& Dostal, P. \\ Department of Process Control, Faculty of Applied Informatics, \\ Tomas Bata University in Zlin, Nad Stranemi 4511, 76272 Zlin, Czech Republic \\ E-Mail: vojtesek@fai.utb.cz
}

\begin{abstract}
Chemical reactors are often characterized by highly nonlinear behaviour. In such cases, the use of conventional control strategies where controllers have fixed parameters can result in poor performance. Knowledge about the static and dynamic properties is a necessary condition for the design of a controller. Unfortunately, measurements on the real system are not always feasible. The only way as to how to obtain static and dynamic behaviour of these systems is through the use of simulations, i.e. experiments on their mathematical model. Steady-state analysis shows the behaviour of the system in the steady state, which can help with the choice of the optimal working point, i.e. the appropriate combination of the input variables, which results in maximal production with minimal cost. On the other hand, the dynamic analysis results in the suitable control strategy. The adaptive approach in this work uses the recursive identification and the polynomial synthesis with the pole-placement method.

(Received in November 2008, accepted in March 2009. This paper was with the authors 1 month for 1 revision.)
\end{abstract}

Key Words: Adaptive Control, Polynomial Approach, Pole-Placement Method, Continuous Stirred Tank Reactor

\section{INTRODUCTION}

Continuous Stirred-Tank Reactors (CSTRs) make up the majority of equipments in the chemical and biochemical industry [1]. Control of such processes is very frequent - e.g. [2], [3] and [4]. This paper is focused on the simulation studies of a CSTR with the Van der Vusse reaction inside [5].

Simulations have big advantages nowadays with the increasing computation power and speed of the computers followed by the decreasing costs. The simulation process usually starts with the modelling of the system. The result of the modelling is the mathematical model of the system, which describes the most important variables and relations between them [6]. The mathematical model of the examined CSTR is described by the set of four nonlinear ordinary differential equations (ODE). The simulation of the steady-state and dynamic behaviour was performed on this mathematical model. The simple iteration method was used for the steady-state analysis [7]. Similarly, the dynamic analysis was performed by RungeKutta's standard method [8]. Results of the steady-state and dynamic analyses are optimal working point and the choice of the External Linear Model (ELM) for the control part.

The adaptive approach [10] used in the control part is based on the choice of External Linear Model (ELM) of the originally nonlinear process, parameters of which are estimated recursively during the control according to the actual state of the system [11]. Although the identification runs in discrete time, the ELM here uses delta models parameters, of which small sampling period are close to the continuous ones [12]. Various types of identifications can be used in the estimation part. The majority of methods are based on the Recursive Least Squares (RLS) method. This method is simple, easily programmable and connected with its 
modifications mainly based on the exponential or directional forgetting [13] provides good identification results.

The controller is designed via polynomial approach which satisfies basic control requirements and moreover, it could be used for systems with negative properties such as non-minimum phase behaviour or for processes with time delays. Connected with pole placement method, it fulfils the requirements of stability, asymptotic tracking of the reference signal and compensation of disturbances [14]. Used control configuration with one degree of freedom (1DOF) has the controller in the feedback part and the reference signal is considered from the ring of step or exponential functions. The resulting controller is strictly proper.

All simulations were performed in the simulation mathematical software MATLAB, version 6.5.1.

\section{MODELLING AND SIMULATION}

\subsection{Mathematical Model}

The reaction inside the reactor is called the Van der Vusse reaction and can be described by the scheme [5] $A \stackrel{k_{1}}{\longrightarrow} B \stackrel{k_{2}}{\longrightarrow} C, 2 A \stackrel{k_{3}}{\longrightarrow} D$.

The mathematical description of the process is very complex and we must introduce some simplifications. In this case, we expect that the reacting mixture is perfectly mixed, all densities, heat capacities and transfer coefficients are constant throughout the reaction. In fact, they are not constant but they usually vary only in a small range, which led us to neglect this variation.

The schematic representation of the model can be found in Fig. 1

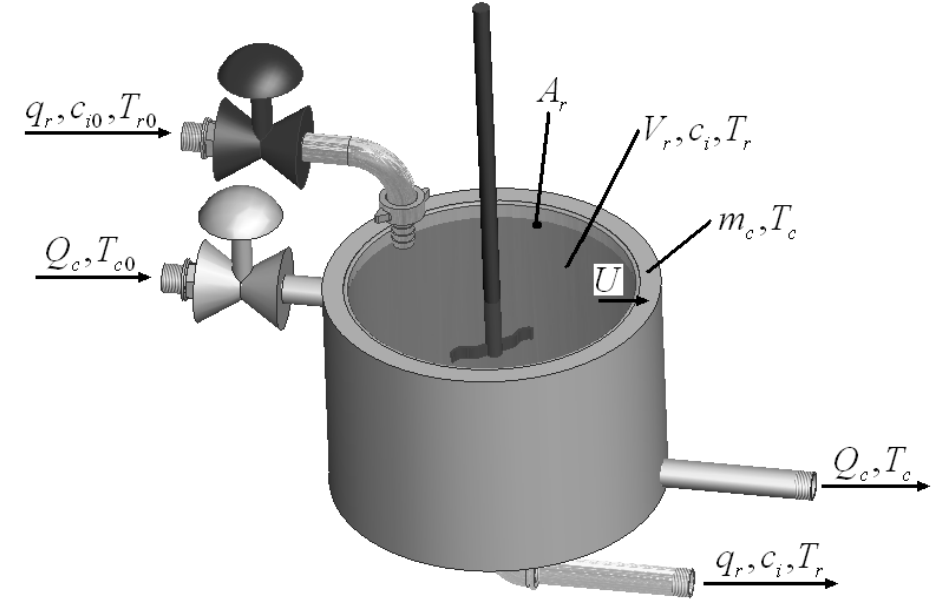

Figure 1: Schematic representation of the Continuous Stirred-Tank Reactor.

With all these simplifications, we can introduce the mathematical model, which is based on two mass and two heat balances inside the reactor:

$$
\begin{gathered}
\frac{d c_{A}}{d t}=\frac{q_{r}}{V_{r}}\left(c_{A 0}-c_{A}\right)-k_{1} c_{A}-k_{3} c_{A}^{2} \\
\frac{d c_{B}}{d t}=-\frac{q_{r}}{V_{r}} c_{B}+k_{1} c_{A}-k_{2} c_{B} \\
\frac{d T_{r}}{d t}=\frac{q_{r}}{V_{r}}\left(T_{r 0}-T_{r}\right)-\frac{h_{r}}{\rho_{r} c_{p r}}+\frac{A_{r} U}{V_{r} \rho_{r} c_{p r}}\left(T_{c}-T_{r}\right)
\end{gathered}
$$




$$
\frac{d T_{c}}{d t}=\frac{1}{m_{c} c_{p c}}\left(Q_{c}+A_{r} U\left(T_{r}-T_{c}\right)\right)
$$

where $c_{A} \geq 0, c_{B} \geq 0$ in virtue of the physical realization.

As can be clearly seen, this mathematical model of the reactor belongs to the class of lumped-parameter nonlinear systems [15] because it is described by a set of ODE. Nonlinearity can be found in reaction rates $\left(k_{j}\right)$, which are described via the Arrhenius law:

$$
k_{j}\left(T_{r}\right)=k_{0 j} \cdot \exp \left(\frac{-E_{j}}{R T_{r}}\right) \text {, for } j=1,2,3
$$

The reaction heat $\left(h_{r}\right)$ in Equation (3) is expressed as:

$$
h_{r}=h_{1} \cdot k_{1} \cdot c_{A}+h_{2} \cdot k_{2} \cdot c_{B}+h_{3} \cdot k_{3} \cdot c_{A}^{2}
$$

\begin{tabular}{|c|c|}
\hline Name of the parameter & Symbol and value of the parameter \\
\hline Volume of the reactor & $V_{r}=0.01 \mathrm{~m}^{3}$ \\
\hline Density of the reacting mixture & $\rho_{r}=934.2 \mathrm{~kg} \cdot \mathrm{m}^{-3}$ \\
\hline Heat capacity of the reacting mixture & $c_{p r}=3.01 \mathrm{~kJ} \mathrm{~kg}^{-1} \cdot \mathrm{K}^{-1}$ \\
\hline Mass of the coolant & $m_{c}=5 \mathrm{~kg}$ \\
\hline Heat capacity of the coolant & $c_{p c}=2.0 \mathrm{~kJ} \mathrm{~kg}^{-1} \cdot \mathrm{K}^{-1}$ \\
\hline Surface of the cooling jacket & $A_{r}=0.215 \mathrm{~m}^{2}$ \\
\hline Heat transfer coefficient & $U=67.2 \mathrm{~kJ} \cdot \mathrm{min}^{-1} \cdot \mathrm{m}^{-2} \cdot \mathrm{K}^{-1}$ \\
\hline Pre-exponential factor for reaction 1 & $k_{01}=2.145 \cdot 10^{10} \min ^{-1}$ \\
\hline Pre-exponential factor for reaction 2 & $k_{02}=2.145 \cdot 10^{10} \min ^{-1}$ \\
\hline Pre-exponential factor for reaction 3 & $k_{03}=1.5072 \cdot 10^{8} \mathrm{~min}^{-1} \mathrm{kmol}^{-1}$ \\
\hline Activation energy of reaction 1 to $R$ & $E_{1} / R=9758.3 \mathrm{~K}$ \\
\hline Activation energy of reaction 2 to $R$ & $E_{2} / R=9758.3 \mathrm{~K}$ \\
\hline Activation energy of reaction 3 to $R$ & $E_{3} / R=8560 \mathrm{~K}$ \\
\hline Enthalpy of reaction 1 & $h_{1}=-4200 \mathrm{~kJ} \mathrm{kmol}^{-1}$ \\
\hline Enthalpy of reaction 2 & $h_{2}=11000 \mathrm{~kJ} \mathrm{kmol}^{-1}$ \\
\hline Enthalpy of reaction 3 & $h_{3}=41850 \mathrm{~kJ} \mathrm{kmol}^{-1}$ \\
\hline Input concentration of compound A & $c_{A 0}=5.1 \mathrm{kmol} \cdot \mathrm{m}^{-3}$ \\
\hline Input temperature of the reacting mixture & $T_{r 0}=387.05 \mathrm{~K}$ \\
\hline
\end{tabular}

Fixed parameters of CSTR [5] are given in Table I.

Table I: Fixed parameters of the reactor.

\subsection{Steady-state Analysis}

Steady-state analysis for stable systems involves computing values of state variables from the steady-state model. There, the variations of all variables and, consequently, the time derivatives in equations of the dynamic process model are equal to zero. In this work, the simple iterative method is used [7]. Since the steady-state equations fulfilling the convergence conditions, this method leads to the exact solution for an appropriate choice of initial iterations, in our case $c_{A}(0)=c_{B}(0)=0 \mathrm{kmol}^{-3}, T_{r}(0)=T_{r 0}=387.05 \mathrm{~K}$ and $T_{c}(0)=293 \mathrm{~K}$. A big advantage of this method is that it is easily programmable which means that there is no need for special functions. 
The steady-state analysis in this case means that the set of ODE (1) - (4) is solved with condition $d(\cdot) / d t=0$. The simple iteration method was used for numerical solving of this problem.

Two steady-state analyses were done - for various heat removal of the cooling liquid, $Q_{c}$, in the range $Q_{c}=<-500 ; 500>\mathrm{kJ}^{-1}$ and various volumetric flow rate of the reacting mixture, $q_{r}$, for values $q_{r}=<0 ; 0.03>\mathrm{m}^{3} \mathrm{~min}^{-1}$.

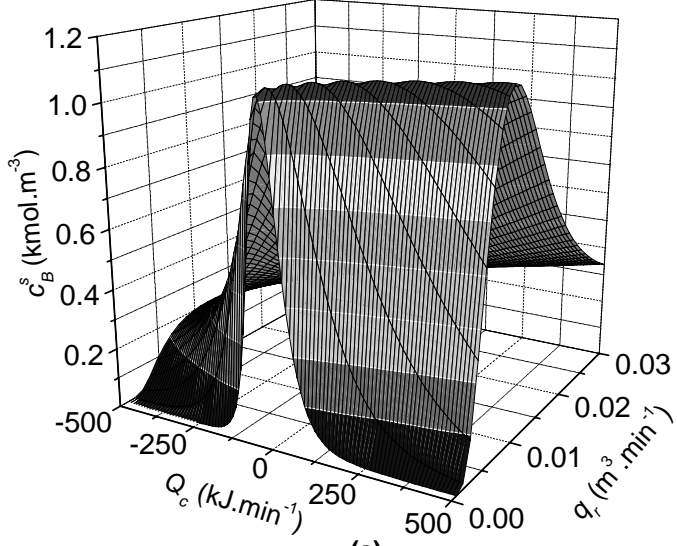

(a)

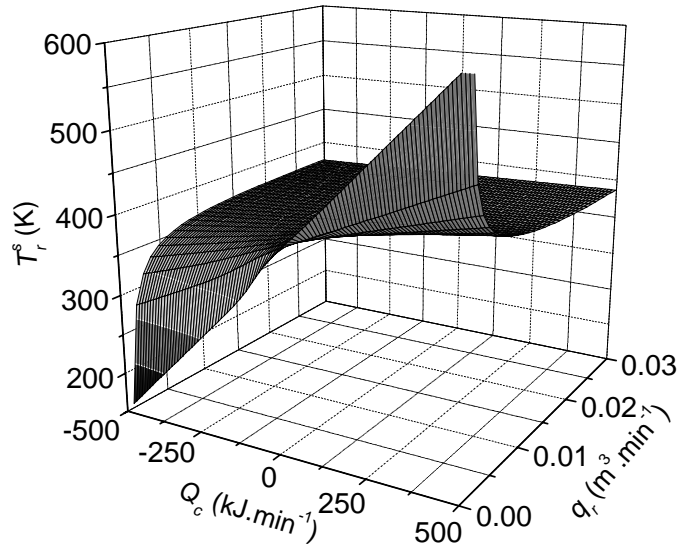

(b)

Figure 2: Steady-state values of the product's concentration $c_{B}{ }^{s}$ (a) and the temperature of the reacting mixture $T_{r}^{s}$ (b) for various heat removals $Q_{c}$ and volumetric flow rate $q_{r}$.

The results presented in Fig. 2 show negative properties of the reactor - especially the course of steady-state values of the concentration $c_{B}{ }^{s}$ has highly nonlinear behaviour. Static analysis usually results in an optimal working point. The maximum of the product's steadystate concentration, ${c_{B}}_{B}{ }^{s}$, was chosen as a criterion for choosing an optimal working point. Concentration ${c_{B}}^{s}$ has its maximum for the volumetric flow rate $q_{r}^{s}=2.365 \cdot 10^{-3} \mathrm{~m}^{3} \mathrm{~min}^{-1}$ and the heat removal $Q_{c}{ }^{s}=-18.56 \mathrm{~kJ} \mathrm{~min}^{-1}$.

Steady-state values for this working point are ${c_{A}}^{s}=2.14 \mathrm{kmol}^{-3},{c_{B}}^{s}=1.09 \mathrm{kmol}^{-3}$, $T_{r}{ }^{s}=387.3 \mathrm{~K}$ and $T_{c}{ }^{s}=386.1 \mathrm{~K}$ and these values are used as initial conditions for the dynamic study.

\subsection{Dynamic Analysis}

Dynamic models of systems with lumped parameters are described by sets of Ordinary Differential Equations (ODEs). Although, there are a lot of methods for finding solutions of ODEs, the popular Runge-Kutta's standard method [8] was used in this work. This method is very often used in the praxis because of its simplicity.

The Runge-Kutta's methods are in some cases build-in functions in mathematical software. For example in MATLAB [9], which is used for simulation in this work, Runge-Kutta's methods are in the functions ode23 (the second order Runge-Kutta formula) or ode45 (the fourth order Runge-Kutta's formula described above). One of advantages of these methods is that they have flexible integration step, which re-computes every step according to the actual computation error.

Dynamic analysis was done for various step changes of the input heat removal of the cooling liquid, $Q_{c}$, which is, for better projection, recomputed to percent via:

$$
u(t)=\frac{Q_{c}(t)-Q_{c}^{s}}{Q_{c}^{s}} \cdot 100 ; \quad[\%] .
$$


Four step changes $\pm 10 \%$ and $\pm 20 \%$ were done. The working point from the steady-state analysis was used and these step changes can be numerically described by $\Delta Q_{c}=3.712(-20 \%), 1.856(-20 \%),-1.856(10 \%),-3.712(20 \%) \mathrm{kJ} \cdot \mathrm{min}^{-1}$.

Output variables $y_{1}$ and $y_{2}$ in the following Fig. 3, illustrate the difference of variables $c_{B}$ and $T_{r}$ from their steady state values, which are in fact initial conditions: $y_{1}(t)=c_{B}(t)-c_{B}{ }^{s}$ and $y_{2}(t)=T_{r}(t)-T_{r}^{s}$. This simplification has only one reason - all graphs start in zero and we can easily estimate time constants, gain etc.

Result of the dynamic analysis show that the output $y_{1}$ has negative properties from the control point of view - non-minimum phase behaviour and changing sign of the gain. On the other hand, that output $y_{2}$ can be approximated by the second order transfer function. This output is chosen as a controlled variable in the control part.
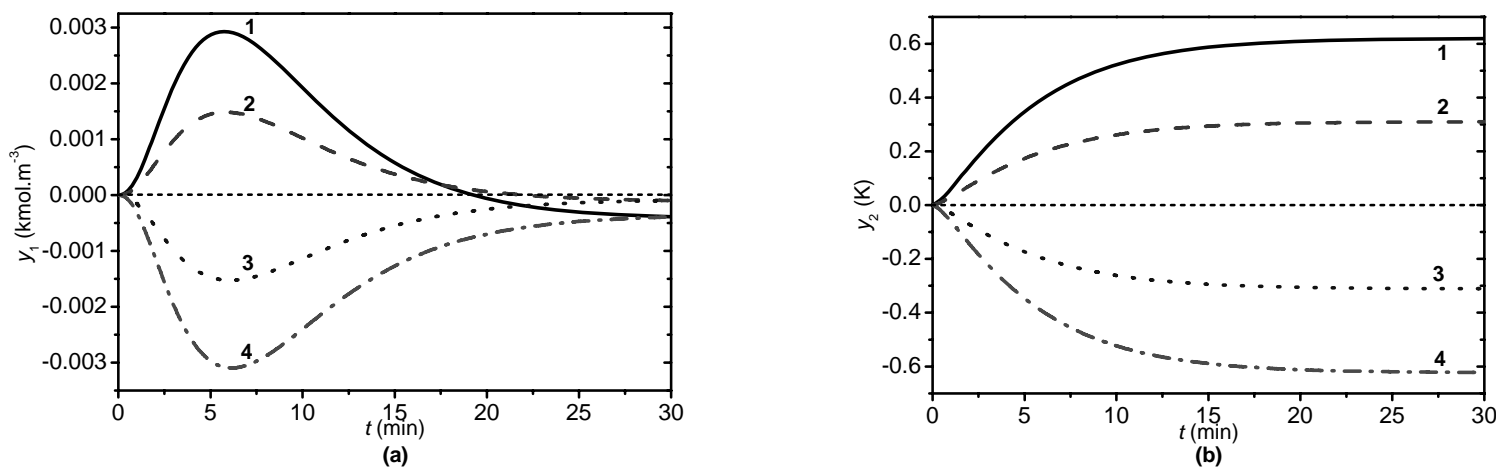

Figure 3: Dynamic analysis of outputs $y_{1}$ (a) and $y_{2}$ (b) for various step changes of the input heat removal $Q_{\mathrm{c}}$.

\section{ADAPTIVE CONTROL}

\subsection{External Linear Model (ELM)}

The CT external linear model (ELM) is chosen on the basis of some preliminary knowledge of the dynamic behaviour of the controlled nonlinear process. This model is described in the time domain by differential equation:

$$
a(\sigma) y(t)=b(\sigma) u(t)
$$

where $\sigma=d / d t$ is the derivative operator and $a, b$ are polynomials in $\sigma$. Considering zero initial conditions, and, using the Laplace transform, the ELM is represented in the complex domain by the transfer function:

$$
G(s)=\frac{Y(s)}{U(s)}=\frac{b(s)}{a(s)}
$$

where $s$ is the complex variable, and both $a$ and $b$ are coprime polynomials in $s$. The transfer function (2) is considered to be proper ( $\operatorname{deg} b \leq \operatorname{deg} a$ ).

Two basic approaches can be used for the identification of the continuous-time ELM. The first method is based on the filtration of input and output signals where the filtered variables have the same properties (in the s-domain) as their non-filtered counterparts, e.g. [16]. Derivatives of filtered signals that are necessary for the parameters estimate of the CT ELM are obtained from differential filters. This method has, however, some drawbacks - the necessity to solve additional differential equations representing the filters and estimate time constants of these filters. 
The second strategy uses an external $\delta$-model of the controlled process with the same structure as a CT model. The basics of $\delta$-models have been described in e.g. [17], [18]. Here, parameters of $\delta$-models can directly be estimated from sampled signals without the necessity to filter them. Moreover, it can be easily proved that these parameters converge to parameters of CT models for a sufficiently small sampling period (compared to the dynamics of the controlled process). A complete description and experimental verification is in [12].

Establishment of the delta operator defined by:

$$
\delta=\frac{\zeta-1}{T_{v}}
$$

where $\zeta$ is the forward shift operator and $T_{v}$ is the sampling period. When the sampling period is shortened, then, the delta operator approaches the derivative operator $\delta$ so that:

$$
\lim _{T_{v} \rightarrow 0} \delta=\sigma
$$

and, the $\delta$-model:

$$
a^{\prime}(\delta) y\left(t^{\prime}\right)=b^{\prime}(\delta) u\left(t^{\prime}\right)
$$

approaches the continuous-time model (8) as shown in [12].

Here, $t^{\prime}$ is the discrete time, and, $a^{\prime}, b^{\prime}$ are polynomials in $\delta$.

With the substitution $t^{\prime}=k-n$ for $k \geq n$, Eq. (12) will be:

$$
\begin{aligned}
\delta^{n} y(k-n)=b_{m}^{\prime} & \delta^{m} u(k-n)+\ldots+b_{1}^{\prime} \delta u(k-n)+b_{0}^{\prime} u(k-n)- \\
& -a_{n-1}^{\prime} \delta^{n-1} y(k-n)-\ldots-a_{1}^{\prime} \delta y(k-n)-a_{0}^{\prime} y(k-n)
\end{aligned} .
$$

Individual elements are:

$$
\begin{aligned}
& \delta^{i} y(k-n)=\sum_{j=0}^{i} \frac{(-1)^{j}}{T_{v}^{i}}\left(\begin{array}{l}
i \\
j
\end{array}\right) y(k-n+i-j), \text { for } i=0,1, \ldots, n \\
& \delta^{l} u(k-n)=\sum_{j=0}^{l} \frac{(-1)^{j}}{T_{v}^{l}}\left(\begin{array}{l}
l \\
j
\end{array}\right) u(k-n+l-j), \text { for } l=0,1, \ldots, m .
\end{aligned}
$$

The individual parts in Eq. (13) can be written as:

$$
\begin{array}{cl}
y_{\delta}(k)=\delta^{n} y(k-n), & u_{\delta}(k-n+m)=\delta^{m} u(k-n), \\
y_{\delta}(k-1)=\delta^{n-1} y(k-n), & u_{\delta}(k-n+m-1)=\delta^{m-1} u(k-n), \\
\vdots & \vdots \\
y_{\delta}(k-n+1)=\delta y(k-n) & u_{\delta}(k-n+1)=\delta u(k-n), \\
y_{\delta}(k-n)=y(k-n) & u_{\delta}(k-n)=u(k-n)
\end{array}
$$

and the regression vector and vector of parameters are then:

$$
\begin{aligned}
& \boldsymbol{\varphi}_{\delta}(k-1)=\left[-y_{\delta}(k-1), \ldots,-y_{\delta}(k-n+1),-y_{\delta}(k-n),\right. \\
& \left.u_{\delta}(k+m-n), u_{\delta}(k+m-1-n), \ldots, u_{\delta}(k-n+1), u_{\delta}(k-n)\right]^{T} . \\
& \boldsymbol{\theta}_{\delta}(k)=\left[a_{n-1}^{\prime}, \ldots, a_{1}^{\prime}, a_{0}^{\prime}, b_{m}^{\prime}, b_{m-1}^{\prime}, \ldots, b_{1}^{\prime}, b_{0}^{\prime}\right]^{T}
\end{aligned}
$$

Parameters of the ELM $\boldsymbol{\theta}_{\boldsymbol{\delta}}$ are then computed from the differential equation:

$$
y_{\delta}(k)=\boldsymbol{\theta}_{\delta}^{T}(k) \cdot \boldsymbol{\varphi}_{\delta}(k-1)+e(k) .
$$

The Resursive Least-Squares (RLS) method [13] was used for the estimation of the vector $\boldsymbol{\theta}_{\delta}$. This method could be described as: 


$$
\begin{gathered}
\varepsilon(k)=y(k)-\boldsymbol{\varphi}_{\delta}^{T}(k) \cdot \hat{\boldsymbol{\theta}}_{\delta}(k-1) \\
\gamma(k)=\left[1+\boldsymbol{\varphi}_{\delta}^{T}(k) \cdot \mathbf{P}(k-1) \cdot \boldsymbol{\varphi}_{\delta}(k)\right]^{-1} \\
\boldsymbol{L}(k)=\gamma(k) \cdot \mathbf{P}(k-1) \cdot \boldsymbol{\varphi}_{\delta}(k) \\
\mathbf{P}(k)=\frac{1}{\lambda_{1}(k-1)}\left[\mathbf{P}(k-1)-\frac{\mathbf{P}(k-1) \cdot \boldsymbol{\varphi}_{\delta}(k) \cdot \boldsymbol{\varphi}_{\delta}^{T}(k) \cdot \mathbf{P}(k-1)}{\lambda_{1}(k-1)+\boldsymbol{\varphi}_{\delta}^{T}(k) \cdot \mathbf{P}(k-1) \cdot \boldsymbol{\varphi}_{\delta}(k)}\right] \\
\hat{\boldsymbol{\theta}}_{\delta}(k)=\hat{\boldsymbol{\theta}}_{\delta}(k-1)+\boldsymbol{L}(k) \varepsilon(k)
\end{gathered}
$$

Several types of exponential forgetting can be used, e.g. RLS with constant exponential forgetting, RLS with increasing exp. forgetting etc. RLS with the changing exp. forgetting is used for parameter estimation here, where the changing forgetting factor $\lambda_{1}$ is computed from:

$$
\lambda_{1}(k)=1-K \cdot \gamma(k) \cdot \varepsilon^{2}(k) \text {. }
$$

The reason why we use this type of identification is because parameters could change during the control and any kind of forgetting could help with estimation where old and unnecessary data affects speed of the identification and its accuracy.

\subsection{Used Adaptive Approach}

As described above, used adaptive approach [10] uses approximation of the originally nonlinear system by the ELM, parameters of which are estimated recursively during the control. Then, the parameters of the adaptive controller are recomputed according to these identified parameters.

The polynomial approach [19] was used for the control synthesis. One of the biggest advantages of the polynomial method compared to the conventional method is that it provides not only relations for computing of the controller's parameters, but the structure of the controller too. Another advantage is that the resulted relations are easily programmable. Polynomials in the numerator and denominator of the transfer function of the controller result from the solution of Diophantine equations. The roots of the characteristic polynomial on the right side of Diophantine equation are then the poles of the closed-loop, which affects the quality of the control. The method of choosing the poles is called the Pole-placement or the Pole-assignment. The polynomial method can be used not only for the configuration with the feedback controller (1DOF configuration), but also for configurations with the feedforward

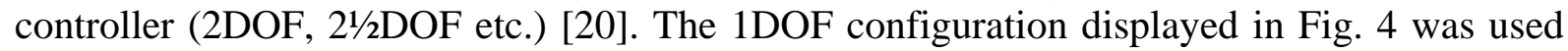
for designing the controller.

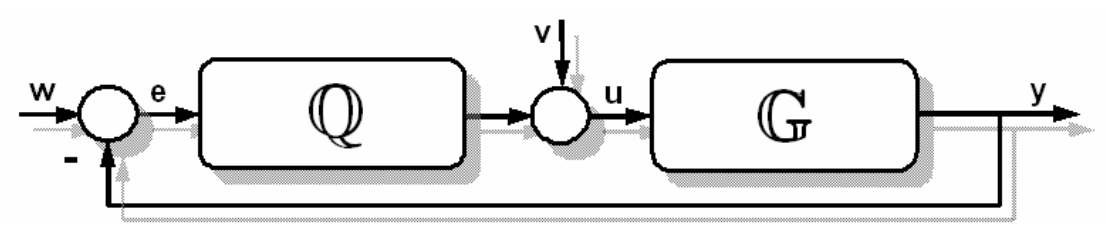

Figure 4: The one degree-of-freedom configuration.

The controlled system is described via transfer function (9) and the feasibility condition is fulfilled if the system is proper, i.e. $\operatorname{deg} a(s) \geq \operatorname{deg} b(s)$.

The transfer function of the controller is:

$$
Q(s)=\frac{q(s)}{s \cdot \tilde{p}(s)}
$$


where polynomials $\tilde{p}(s)$ and $q(s)$ are coprime polynomials with the condition of properness $\operatorname{deg} p(s) \geq \operatorname{deg} q(s)$. Degrees of the polynomials are given as:

$$
\operatorname{deg} q(s)=\operatorname{deg} a(s), \operatorname{deg} \tilde{p}(s) \geq \operatorname{deg} a(s)-1 .
$$

Parameters of these polynomials are computed from Diophantine equation:

$$
a(s) \cdot s \cdot \tilde{p}(s)+b(s) \cdot q(s)=d(s) \text {. }
$$

The polynomial $d(s)$ on the right side of (27) is an optional stable polynomial. Roots of this polynomial are called poles of the closed-loop and their position affects quality of the control. Block $Q$ ensures stability, asymptotic tracking of the reference signal and load disturbance attenuation. The degree of the polynomial $d(s)$ is in this case:

$$
\operatorname{deg} d(s)=\operatorname{deg} a(s)+\operatorname{deg} \tilde{p}(s)+1 .
$$

The simplest way how to choose roots of polynomial $d(s)$ is selection of double, triple etc. multiple roots:

$$
d(s)=(s-\alpha)^{m} ; d(s)=\left(s-\alpha_{1}\right)^{m / 2} \cdot\left(s-\alpha_{2}\right)^{m / 2}, \ldots
$$

A choice of these roots needs some a priori information about the system's behaviour. It is good to connect poles with the parameters of the system for example via spectral factorization. Polynomial $d(s)$ is then divided into two parts $-m(s)$ and $n(s)$, so:

$$
d(s)=m(s) \cdot n(s)
$$

where polynomial $n(s)$ is computed from the spectral factorization of polynomial $a(s)$ in the denominator of the transfer function $G(s)$ described in (9):

$$
n^{*}(s) \cdot n(s)=a^{*}(s) \cdot a(s)
$$

and polynomial $m(s)$ is a stable one:

$$
m(s)=\left(s-\alpha_{i}\right)^{\operatorname{deg} d-\operatorname{deg} n}
$$

where $\alpha_{i}$ are (deg $d-\operatorname{deg} n$ )-optional stable (i.e. negative) roots, usually called poles of the control system. A disadvantage of this method is that it still has an uncertainty in polynomial $m(s)$.

\subsection{Simulation Results}

Based on the dynamic study, the step change of the heat removal of the cooling, $Q_{c}$, was chosen as an input (control) variable $u(t)$ and the temperature of the reacting mixture, $T_{r}$, again related to its initial condition (steady-state value) was used as an output (controlled) variable $y(t)$. This output $y(t)$ can be represented according to Fig. 3 by the second order transfer function with relative order one:

$$
G(s)=\frac{b(s)}{a(s)}=\frac{b_{1} s+b_{0}}{s^{2}+a_{1} s+a_{0}}
$$

The parameters of polynomials $a(s)$ and $b(s)$ in (33) are estimated recursively during the control with the use of RLS method with changing exponential forgetting. The initial values are: $K=0.001$ and parameters $\gamma(0)=0, \varepsilon(0)=0$.

Degrees of the polynomials $q(s)$ and $\tilde{p}(s)$ controller $Q(s)$ and polynomial $d(s)$ in the 1DOF configuration are for the second order transfer function (33): 


$$
\begin{gathered}
\operatorname{deg} d(s)=\operatorname{deg} a(s)+\operatorname{deg} \tilde{p}(s)+1=2+1+1=4 \\
\quad \operatorname{deg} q(s)=\operatorname{deg} a(s)=2 \\
\operatorname{deg} \tilde{p}(s) \geq \operatorname{deg} a(s)-1 \Rightarrow \operatorname{deg} \tilde{p}(s)=2-1=1
\end{gathered}
$$

which means that the transfer function of the controller $Q(s)$ is:

$$
\tilde{Q}(s)=\frac{q(s)}{s \cdot \tilde{p}(s)}=\frac{q_{2} s^{2}+q_{1} s+q_{0}}{s \cdot\left(s+p_{0}\right)}
$$

where the parameters of polynomials $q(s)$ and $\tilde{p}(s)$ are computed from the Diophantine equation (27) by the method of comparison of the coefficients.

The estimated output is recomputed recursively from differential equation:

$$
y_{\delta}(k)=\boldsymbol{\theta}_{\delta}^{T}(k) \cdot \boldsymbol{\varphi}_{\delta}(k-1)
$$

where the vector of the parameters, $\boldsymbol{\theta}_{\delta}$, and the data vector, $\boldsymbol{\varphi}_{\delta}$, are according to the order of ELM (36):

$$
\boldsymbol{\theta}_{\delta}(k)=\left[a_{1}^{\prime}, a_{0}^{\prime}, b_{1}^{\prime}, b_{0}^{\prime}\right]^{T} ; \quad \boldsymbol{\varphi}_{\delta}(k-1)=\left[-y_{\delta}(k-1),-y_{\delta}(k-2), u_{\delta}(k-1), u_{\delta}(k-2)\right]^{T}
$$

where:

$$
\begin{gathered}
y_{\delta}(k)=\frac{y(k)-2 y(k-1)+y(k-2)}{T_{v}^{2}}, y_{\delta}(k-1)=\frac{y(k-1)-y(k-2)}{T_{v}}, y_{\delta}(k-2)=y(k-2) \\
u_{\delta}(k-1)=\frac{u(k-1)-u(k-2)}{T_{v}}, \quad u_{\delta}(k-2)=u(k-2)
\end{gathered}
$$

Three simulation studies were done for different values of parameter $\alpha_{i}=0.05,0.1$ and 0.4, which represent the position of the double root. Polynomial $d(s)$ on the right side of the Diophantine equation can be divided into two polynomials: $d(s)=m(s) \cdot n(s)$, where polynomial $n(s)$ is computed by the spectral factorization of polynomial $a(s): n^{*}(s) \cdot n(\mathrm{~s})=$ $a^{*}(s) \cdot a(s)$. The degree of polynomial $n(s)$ is equal to deg $a(s)$, i.e.:

$$
n(s)=s^{2}+n_{1} s+n_{0} \Rightarrow n_{0}=\sqrt{a_{0}^{2}} ; n_{1}=\sqrt{a_{1}^{2}+2 n_{0}-2 a_{0}} \text {. }
$$

The second part of polynomial $d(s)$, stable polynomial $m(s)$ was chosen $m(s)=\left(s+\alpha_{i}\right)^{\operatorname{deg} \mathrm{d}-\operatorname{deg} \mathrm{n}}=\left(s+\alpha_{i}\right)$ which results in one double root where $\alpha_{i}>0$.

The working point is $c_{A 0}=5.1 \mathrm{kmolm}^{-3}, T_{r 0}=387.05 \mathrm{~K}, q_{r}^{s}=2.365 \cdot 10^{-3} \mathrm{~m}^{3} \mathrm{~min}^{-1}$ and $Q_{c}{ }^{s}=-18.56 \mathrm{~kJ} \mathrm{~min}^{-1}$. The initial values for identification are $\boldsymbol{\theta}_{\delta}=[0.1,0.1,0.1,0.1]^{T}$ and covariance matrix $\mathbf{P}$ has size $4 \times 4$ and $1 \cdot 10^{6}$ on the main diagonal. The quality of control was evaluated by the quality criteria $S_{u}$ and $S_{y}$ computed for a time interval as:

$$
S_{u}=\sum_{i=2}^{N}(u(i)-u(i-1))^{2}[-] ; \quad S_{y}=\sum_{i=1}^{N}(w(i)-y(i))^{2}\left[K^{2}\right] \text {, for } N=\frac{T_{f}}{T_{v}} .
$$

Where, $T_{f}$ is the time of the simulation, in our case $450 \mathrm{~min}$, the sampling period is $T_{v}=0.3 \mathrm{~min}$ and three changes of the reference signal (wanted value) were done. The action value (input signal) is limited due to technological reasons $u(t)=<-75 ;+75>\%$ of $Q_{c}{ }^{s}$.

Fig. 5 shows good control results except of the beginning of the control, which is caused by the identification, because the controller has not enough initial information about the system and needs some time for tuning. Figures show that the output response is quicker with increasing value of parameter $\alpha_{i}$. On the other hand, there can be some overshoots of the output variable and the course of the input variable, $u(t)$, is not so smooth for bigger values of $\alpha_{\text {i }}$ 

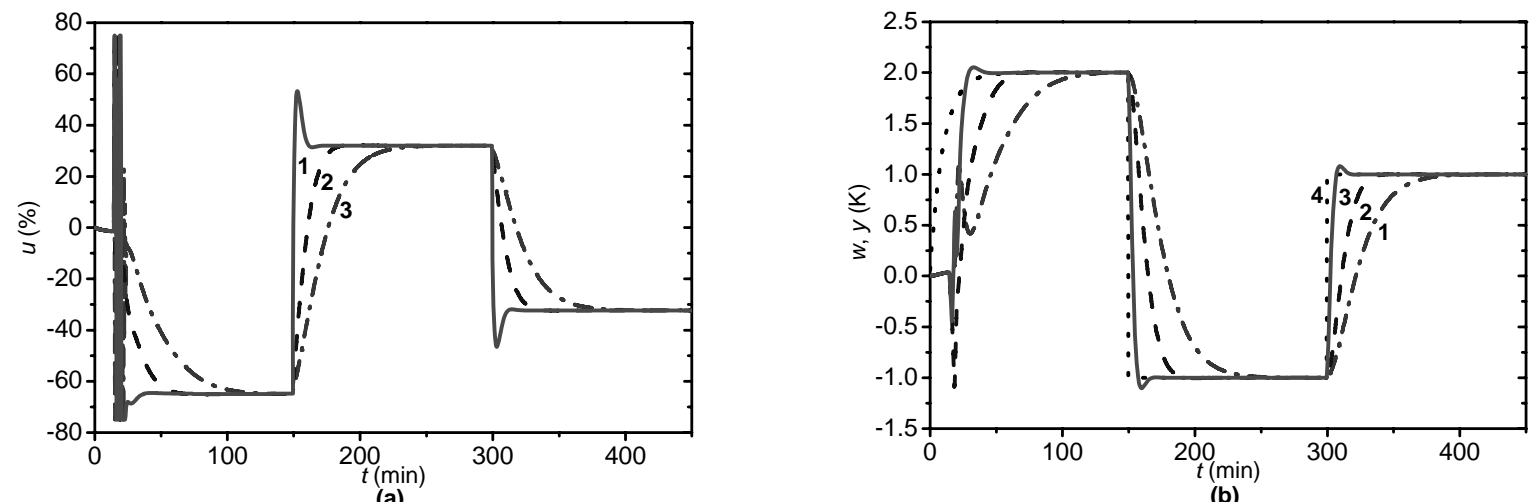

Figure 5: The course of the input variable $u(t)$ (a), the output variable $y(t)$ (b) and the reference signal $w(t)$ (4) for different position of the root $\alpha_{i}=0.05$ (1), 0.1 (2) and 0.4 (3).

The course of identified parameters $a^{\prime}{ }_{0}, a^{\prime}{ }_{1}, b^{\prime}{ }_{0}$ and $b^{\prime}{ }_{1}$ in Figs. 6 and 7 shows that the used recursive identification has no problem with parameter estimation during the control except for the very beginning, as mentioned above. This disadvantage could be overcome with the use of simple proportional controller for some initial time or by replacing of the ordinary step function for the reference signal $w(t)$ by some type of exponential function, as it was used in our case where the first change is described by $w(t)=2 \cdot[1-\exp (-0.1 \cdot t)]$.
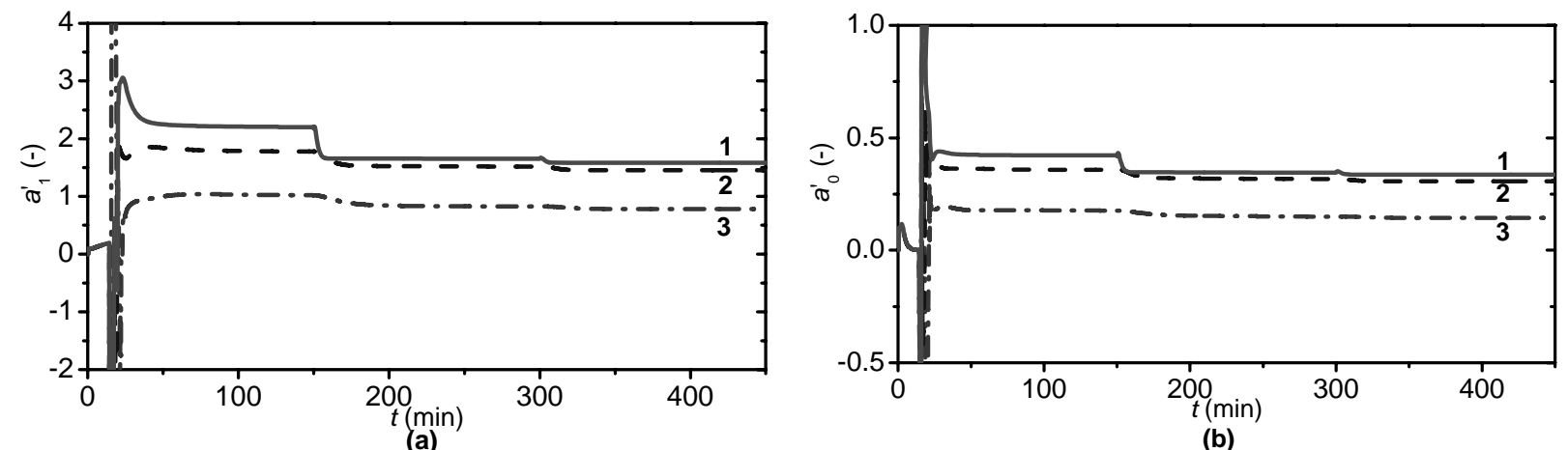

(b)
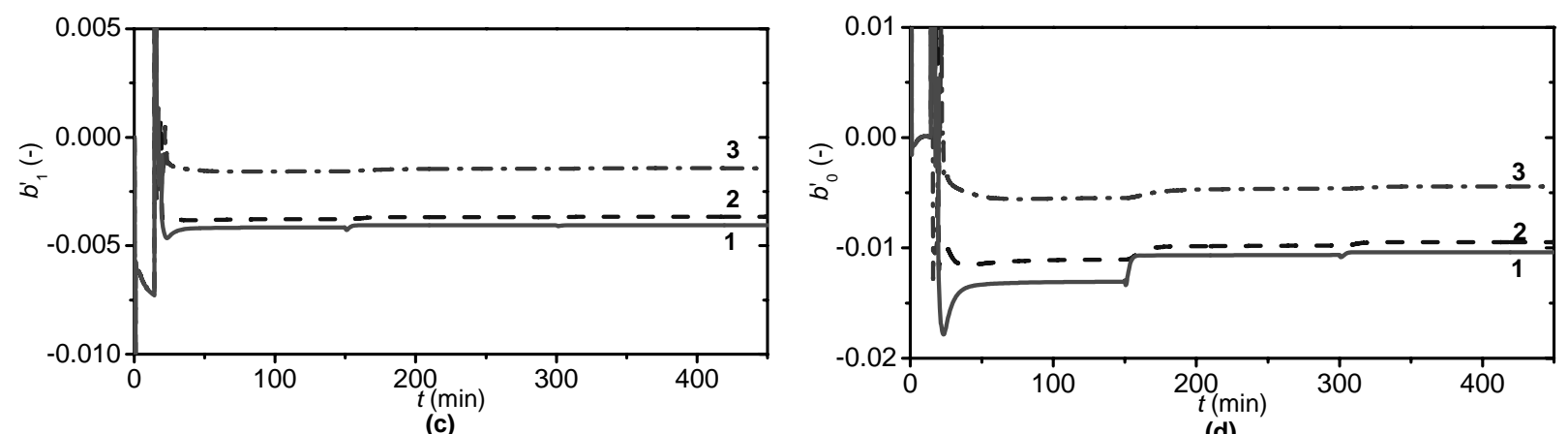

(d)

Figure 6: The course of estimated parameters $a^{\prime}{ }_{0}(\mathrm{a}), a^{\prime}{ }_{1}(\mathrm{~b}), b^{\prime}{ }_{0}$ (c) and $b^{\prime}{ }_{1}$ (d) for different position of the parameter $\alpha_{i}=0.05$ (1), 0.1 (2) and 0.4 (3).

Table II presents the results of control from the control quality point of view, according to Eq. (40). The best result from the output point of view (parameter $S_{y}$ ) is for the parameter $\alpha_{i}=$ 0.4. On the other hand, more considerate to the input variable is the controller with the parameter $\alpha_{i}=0.1$.

As mentioned above, the nominated adaptive controller can deal with disturbance attenuation, which is proved in the following figure. The simulation was done again for 
various values of parameter $\alpha_{i}=0.05,0.1$ and 0.4 , the sampling period was $T_{v}=0.3 \mathrm{~min}$, the simulation time 500 min with one step change $w(t)=2 \cdot[1-\exp (-0.1 \cdot t)]$. Three types of disturbances, two in the input $\left(v_{1}(t)=+3 \%\right.$ step change of the input concentration $c_{A 0}$ in time $t=150 \mathrm{~min}, v_{2}(t)=-0.5 \mathrm{~K}$ step change of the input temperature $T_{r 0}$ in time $t=250 \mathrm{~min}$ ) and one on the output $\left(v_{3}(t)=0.5 \mathrm{~K}\right.$ step change of the output temperature $T_{r}$ in time $t=400 \mathrm{~min}$ ), are injected to the system during this time.

Table II: The results of control from the control quality criteria $S_{u}, S_{y}$ point of view.

\begin{tabular}{|c|c|c|}
\hline & $S_{u}[-]$ & $S_{y}\left[\mathrm{~K}^{2}\right]$ \\
\hline$\alpha_{i}=0.05$ & 60457 & 975.19 \\
$\alpha_{i}=0.1$ & 32151 & 590.24 \\
$\alpha_{i}=0.4$ & 62933 & 198.81 \\
\hline
\end{tabular}

As can be seen from following figure, presence of the integration part in the controller ensures full attenuation of the disturbances on the input or output, respectively. All three disturbances are suppressed by the used adaptive controller. The only difference is in the speed of control and disturbance attenuation. The response with the lowest value of $\alpha_{i}=0.05$ results in the slowest course of the output variable and the disturbance is suppressed slowly, while the biggest value, $\alpha_{i}=0.4$ represses the influence of the disturbance faster but with a small overshoot of the output variable at the very beginning.

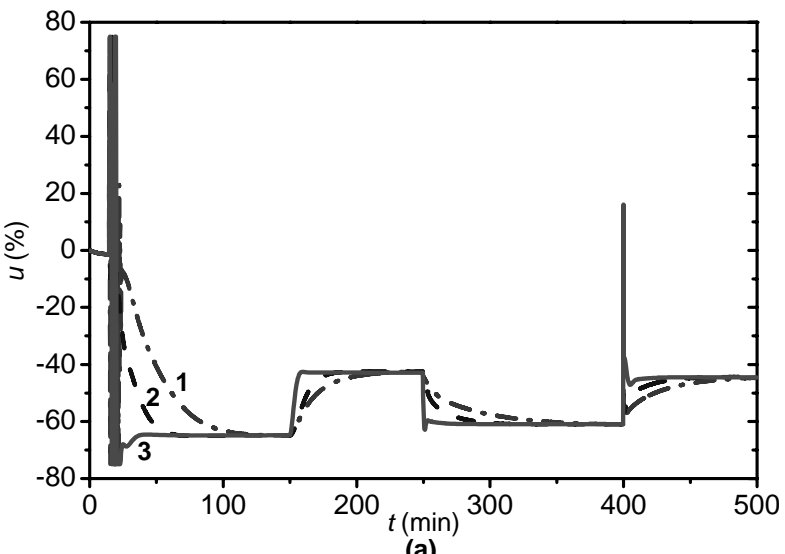

(a)

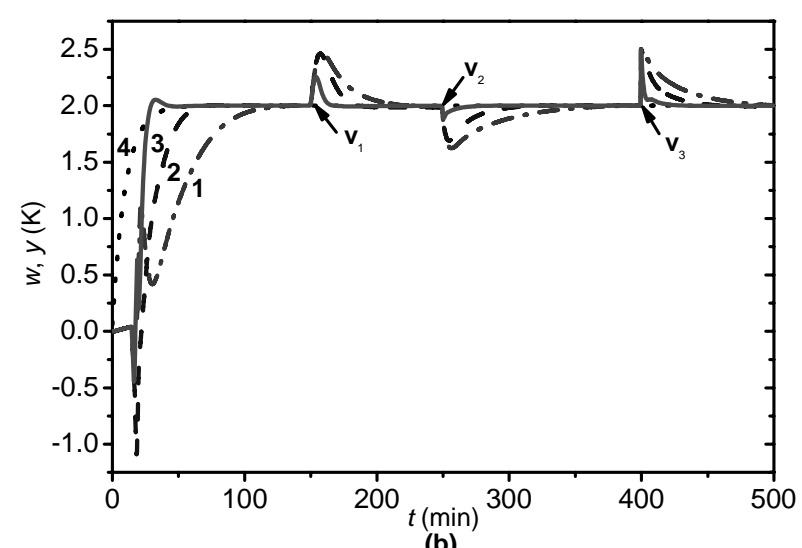

(b)

Figure 7: The course of the input variable $u(t)$, the output variable $y(t)$ and the reference signal $w(t)$ (4) for different position of the root $\alpha_{i}=0.05$ (1), 0.1 (2) and 0.4 (3) and for three disturbances $v_{1}(t), v_{2}(t)$ and $v_{3}(t)$.

\section{CONCLUSIONS}

The main objective of this work was to show the process from simulations of steady-state and dynamic analysis to simulations of adaptive control of CSTR with so called Van der Vusse reaction inside. This type of chemical reactor is a typical representative of nonlinear process, which makes them difficult to control.

However, adaptive control used for controlling produced good control results, although the model has negative control properties such as nonlinearity, non-minimum phase behaviour or changing sign of gain. A problem with the used adaptive approach can be found at the beginning of control. The controller does not have sufficient information about the controlled 
system, which results in inappropriate control responses and overshoots. However, the control response after the second and higher step change is usually much better.

This disadvantage could be reduced with the use of exponential function for the reference signal instead of the ordinary step function. The optional tuning parameter in the poleplacement method with spectral factorization is position of the pole (root) $\alpha_{i}$. The increasing value of this parameter results in quicker responses but overshoots of the output variable, as it is shown in the practical part. Proposed adaptive controllers have good results of the control and fulfilled basic control requirements such as the stability, the reference signal tracking and disturbance attenuation.

The future work will be focused on the application of these simulation and control studies on the real CSTR as a verification of the simulation results credibility.

\section{ACKNOWLEDGEMENTS}

This work was supported by the Ministry of Education of the Czech Rep. under grant No. MSM 7088352101.

\section{REFERENCES}

[1] Smith, R. M. (2005). Chemical Process: Design and Integration, John Wiley \& Sons, Chichester

[2] Corriou, J.-P. (2004). Process control. Theory and applications, Springer-Verlag, London

[3] Ingham, J.; Dunn, I. J.; Heinzle, E.; Přenosil, J. E. (2000). Chemical Engineering Dynamics. An Introduction to Modelling and Computer Simulation, VCH Verlagsgesellshaft, Weinheim

[4] Hourfar, F.; Salahshoor, K. (2008). Adaptive Control of CSTR Using Feedback Linearization Based on Grey-Box Modeling, Proceedings of IEEE International Conference on Networking, Sensing and Control - ICNSC 2008, Sanya, China

[5] Chen, H.; Kremling, A.; Allgöwer, F. (1995). Nonlinear Predictive Control of a Benchmark CSTR, Proceedings of $3^{\text {rd }}$ European Control Conference, Rome, Italy

[6] Babu, B. V. (2004). Process Plant Simulation, Oxford University Press

[7] Meerschaert, M. (2007). Mathematical Modeling, Academic Press, London

[8] Johnston, R. L. (1982). Numerical Methods, John Wiley \& Sons, New York

[9] Mathews, J. H.; Fink, K. K. (2004). Numerical Methods Using Matlab, Prentice-Hall, Upper Saddle River

[10] Astrom, K. J.; Wittenmark, B. (1995). Adaptive control, Addison Wesley, Reaching

[11] Bobál, V.; Böhm, J.; Fessl, J.; Macháček, J. (2005). Digital Self-tuning Controllers. Algorithms, Implementation and Applications, Springer-Verlag, London

[12] Stericker, D. L.; Sinha, N. K. (1993). Identification of continuous-time systems from samples of input-output data using the $\delta$-operator, Control-Theory and Advanced Technology, Vol. 9, 113125

[13] Fikar, M.; Mikleš, J. (1999). System identification (in Slovak), STU Bratislava

[14] Kučera, V. (1993). Diophantine equations in control - A survey, Automatica, Vol. 29, 1361-1375

[15] Wellstead, P. E. (2000). Introduction to physical system modelling, Academic Press, London

[16] Wahlberg, B. (1990). The effects of rapid sampling in system identification, Automatica, Vol. 26, 167-170

[17] Middleton, R. H.; Goodwin, G. C. (1990). Digital Control and Estimation - A Unified Approach, Prentice Hall, Englewood Cliffs

[18] Mukhopadhyay, S.; Patra, A. G.; Rao, G. P. (1992). New class of discrete-time models for continuos-time systems, International Journal of Control, Vol. 55, 1161-1187

[19] Kučera, V. (1991). Analysis and Design of Discrete Linear Control Systems, Prentice-Hall, London

[20] Grimble, M. J. (1994). Robust industrial control. Optimal design approach for polynomial systems, Prentice Hall, London 